\title{
Rapid Detection of Proliferative Bacteria by Electrical Stimulation
}

Conor LA Edwards, Dmitry Malyshev, James P Stratford and Munehiro Asally*

School of Life Sciences, University of Warwick, Coventry, CV4 7AL, The United Kingdom

*For correspondence: m.asally@warwick.ac.uk

\begin{abstract}
[Abstract] Detecting live bacteria is an important task for antimicrobial susceptibility testing (AST) in the medical sector and for quality-monitoring in biological industries. Current methods for live-bacteria detection suffer limitations in speed or sensitivity. In a recent paper, we reported that electrical response dynamics in membrane potential enable single-cell rapid detection of live bacteria. The electrical response can be observed within a minute after electrical stimulation. Thus, it has potential in accelerating AST and the monitoring of biological samples. This method also enables experiments for biophysical and microbiological investigations into bacterial electrophysiology. With the hope that more researchers, scientists and engineers will use electrical stimulation for their assays, here we detail each step of the electrical stimulation experiment.
\end{abstract}

Keywords: Bacterial electrophysiology, Membrane potential, Bacterial detection, Anti-microbial resistance (AMR), Cell vitality assay, Cell biophysics

[Background] From fundamental microbiology research to antimicrobial susceptibility testing (AST), quantifying the number of live bacteria is an important task. When it comes to detecting live bacteria, the colony forming unit (CFU) assay, dating from the $19^{\text {th }}$ century, is still the gold standard due to its reliability and sensitivity. However, the CFU assay has a major drawback; it requires 1-3 days of incubation. To overcome this limitation, developments in rapid bacterial detection technology has flourished in recent years (lqbal et al., 2000; Ahmed et al., 2014). Examples of recently developed technologies are based on impedance spectroscopy, Raman spectroscopy or detection of biomolecules (e.g., ATP, DNA). Nevertheless, overcoming the tradeoff between speed, accuracy and robustness remains a challenge.

Membrane potential indicators are proven useful for distinguishing live and dead bacteria by flow cytometry and fluorescence microscopy (Sträuber and Müller, 2010). However, this method is technically challenging because it requires a careful calibration for bacterial species/strains, media conditions, indicator concentrations, light sources and detectors. In a recent study, we showed that such limitations can be overcome using optical measurements of the electrical response dynamics of membrane potential under stimulation by an external electrical field (Stratford et al., 2019). The responses of proliferative and inhibited cells were distinguishable within a minute; hence, it substantially shortens the time required for a live bacterial assay. Simulations of a phenomenological mathematical model and biophysical understanding suggested that this technology is applicable for different microbial species and antimicrobial treatments. This means that by comparing the electrical dynamics of unperturbed and antimicrobial-treated cells, it may be possible to accelerate AST. An important next step is examining 
how widely this technology can be used with environmental and pathogenic microbes. This protocol will assist conducting electrical stimulation experiments for AST and live bacteria detection assays with various bacterial species and strains as well as with various antimicrobial treatments.

In addition to its fundamental role in cell proliferation, membrane potential mediates bacterial electrical signaling in biofilms and during the processes of sporulation, mechano-sensation and cell division (Strahl and Hamoen, 2010; Prindle et al., 2015; Bruni et al., 2017; Sirec et al., 2019, Benarroch and Asally, 2020). Membrane potential is also associated with antibiotic resistance (Damper and Epstein, 2010). While these recent studies are beginning to garner attention, membrane potential remains largely overlooked in the field of microbiology. We suspect this oversight is partially due to the technical difficulties associated with experimentally controlling membrane potential. To this end, this protocol will also assist biophysical and microbiological investigations into bacterial electrophysiology.

\section{Materials and Reagents}

Notes:

a. The following chemicals are interchangeable with appropriate replacements, per the investigator's discretion.

b. All these ingredients can be stored in dry conditions at room temperature.

1. Stainless steel mounting wafer

2. Copper wires

3. Parafilm

4. $22 \times 22 \mathrm{~mm}$ glass slip

5. Microscope Coverslips

6. Bacterial samples

7. Titanium-Gold Alloy

8. Difco ${ }^{T M}$ Agar, Granulated (VWR, BD Biosciences, catalog number: 90000-786)

9. L-Glutamic acid monosodium hydrate (Sigma-Aldrich, catalog number: G1626)

10. Glycerol $99 \%$ purity (Fisher Chemical, catalog number: G/0650/17)

11. 3-(N-morpholino)propanesulfonic acid (MOPS) (Sigma-Aldrich, catalog number: M9381)

12. Potassium Phosphate dibasic Trihydrate, $\mathrm{K}_{2} \mathrm{HPO}_{4} \cdot 7 \mathrm{H}_{2} \mathrm{O}$ (Sigma-Aldrich, catalog number: 431478)

13. Potassium Dihydrogen Orthophosphate, $\mathrm{KH}_{2} \mathrm{PO}_{4}$ (Fisher Chemical, catalog number: 7778-77$0)$

14. D-Glucose (Sigma-Aldrich, catalog number: D9434)

15. Ammonium Chloride, $\mathrm{NH}_{4} \mathrm{Cl}$ (Fisher Chemical, catalog number: 12125-02-9)

16. Calcium Chloride, $\mathrm{CaCl}_{2} \cdot 2 \mathrm{H}_{2} \mathrm{O}$ (Fisher Chemical, catalog number: 10035-04-8)

17. Magnesium Chloride, $\mathrm{MgCl}_{2}$ (Fisher Chemical, catalog number: 7791-18-6)

18. Iron (III) Chloride, $\mathrm{FeCl}_{3} \cdot 6 \mathrm{H}_{2} \mathrm{O}$ (Fisher Chemical, catalog number: 10025-77-1) 
19. Manganese Chloride, $\mathrm{MnCl}_{2}$ (Sigma-Aldrich, catalog number: 244589)

20. Zinc Chloride, $\mathrm{ZnCl}_{2}$ (Fisher Chemical, catalog number: 7646-85-7)

21. Thiamine Hydrochloride (Sigma-Aldrich, catalog number: T4625)

22. Thioflavin T (Sigma Aldrich, catalog number: T3516)

23. $\mathrm{NaOH}$

24. LB Broth (Fisher Scientific, catalog number: BP1426-2)

25. $0.5 \mathrm{M}$ potassium phosphate buffer ( $\mathrm{pH} 7)$ (see Recipes)

26. MOPS buffer (see Recipes)

27. Modified MSgg (see Recipes)

\section{Equipment}

Note: The list of the specific equipment used in Stratford et al., 2019 can be found in Tables 1-2.

\section{Essential Equipment}

1. Inverted Fluorescence Microscope

2. Microscope Incubation Chamber

3. Temperature Controller

4. Fluorescent Light Source

5. Arbitrary Function Generator

6. Bespoke electrode dishes (The bill of materials is in Table 3)

7. Relay Circuit (The diagram is in Figure 4)

8. Electron Beam Vapor Deposition System or equivalent alloy deposition method Note: Items \#5-8 are not necessary with CytePulse method.

\section{Supplementary Equipment}

1. $\mathrm{pH}$ meter or testing strips

2. Weighing scales

3. Shaking incubator

4. Adjustable volume pipettes ranging from $0.1 \mu \mathrm{l}$ to $1,000 \mu \mathrm{l}$

5. Measuring cylinder or serological pipettes ranging from 5-100 ml

6. Scalpel

7. Tweezers

8. Bunsen Burner 
Table 1. List of equipment for Example Essential Equipment

\begin{tabular}{|c|c|}
\hline \multicolumn{2}{|l|}{ Essential Equipment } \\
\hline Main text Reference & Founding Study Equipment \\
\hline Multi-stage Fluorescence Microscope & $\begin{array}{l}\text { Leica DMi8 microscope with Leica CTR advanced } \\
\text { electronics box (Leica) }\end{array}$ \\
\hline Incubation Chamber & PeCon GmbH (PeCon) \\
\hline Temperature Controller & TempController 2000-2 heater unit (Pecon) \\
\hline Fluorescent Light Source & $\begin{array}{l}\text { Lumencor SOLA SE Light Engine light source } \\
\text { (Lumencor) }\end{array}$ \\
\hline Arbitrary Function Generator & $\begin{array}{l}\text { Tektronix AFG1062 function generator (RS, } \\
\text { catalog number: 898-6858) }\end{array}$ \\
\hline Bespoke electrode dishes & Construction Process: see Procedure A \\
\hline & Bill of materials: Table 3 \\
\hline CytePulse consumables. & CytePulse consumables (Cytecom) \\
\hline Relay Circuit & $\begin{array}{l}\text { Bill of materials: Table } 3 . \\
\text { Circuit Diagram: Figure } 4\end{array}$ \\
\hline Electron vapor deposition system & $\begin{array}{l}\text { Custom-built model II2000EB (Scientific Vacuum } \\
\text { Systems) }\end{array}$ \\
\hline
\end{tabular}

Table 2. List of equipment for Example Supplementary Equipment

\section{Supplementary Equipment}

\section{Main text Reference}

pH Meter

Weighing Scales

Shaking Incubator

Adjustable Volume Pipettes

Spectrophotometer

\section{Founding Study Equipment}

$\mathrm{pH}$ Meter (Fisher-Scientific, catalog number: AE150)

Analytical balance (Fisherbrand, catalog number: PS100)

Shaking incubator (Labnet, catalog number: 311DS)

Adjustable Volume Pipettes (Eppendorf, Research Plus)

Jenway 7305 Spectrophotometer 
Table 3. Bespoke electrode dish bill of materials

\begin{tabular}{llcc}
\hline Component & Manufacturer & $\begin{array}{c}\text { Manufacturer } \\
\text { Part Number }\end{array}$ & Quantity \\
& & HBST-5040 & 4 \\
\hline Glass-bottom microscope dish & WillCo Wells & $2457-\mathrm{H}$ & 1 \\
Araldite two-part epoxy & Araldite & SP515-50 & 50 \\
Neodynium Magnets & Magnets4U & NA & 1 \\
Stainless-steel mask & Laser & & \\
& micromachining & NA & 4 \\
M6 Flat Repair washer & Singularity & & \\
& supplies & A000073 & 1 \\
Arduino Uno (REV 3) & Arduino & $291-9704$ & 7 \\
Relay & RS PRO & 1725656 & 4 \\
Phoenix Contact & Phoenix Contact & $526-5864$ & 4 \\
Female BNC Connector & RS PRO & LR1F1M0 & 7 \\
Resistor 1M 2 & TE Connectivity & $01-0021$ & 1 \\
Prototyping Breadboard & Vero Technologies & $625-7252$ & 7 \\
10-way IDC connector (Male) & RS PRO & $625-7369$ & 4 \\
10-way IDC connector & RS PRO & & 1 \\
(Female) & & $360-093$ & 1 \\
10-way Ribbon Cable Reel & RS PRO & $251-8121$ & 3 \\
2.54 mm pitch Header Pins & RS PRO & $791-6463$ & 1 \\
Breadboard Jumper Wire & RS PRO & & \\
\hline
\end{tabular}

\section{Software}

1. Microscope Control Software (e.g., MetaMorph, micro-manager [micro-manager.org/])

2. Arduino IDE (arduino.cC) (not necessary with Cytepulse method)

3. Image processing software (e.g., Fiji/lmageJ (fiji.sc) (Schindelin et al., 2012))

4. Data processing software (e.g., Matlab, Anaconda Python/R Distribution [anaconda.com]).

\section{Procedure}

For those using the bespoke method, as in Stratford et al. (2019) all steps must be followed. For those using the CytePulse system (Cytecom Ltd), skip to Procedure C "Sample preparation" section. Also, of note, those steps demarcated with an asterisk $\left(^{*}\right)$ in the procedure only pertain to those following the bespoke method. If using the CytePulse product and consumables, disregard these steps.

A. Bespoke electrode dish construction

The following methods are based on the use of an electron beam vapor deposition system. Different 
methods may vary between different constructions. Figure 2 provides an illustrative instruction.

1. Construct a negative stainless-steel mask (Figure 1). This is done by a Laser Manufacturing Company.

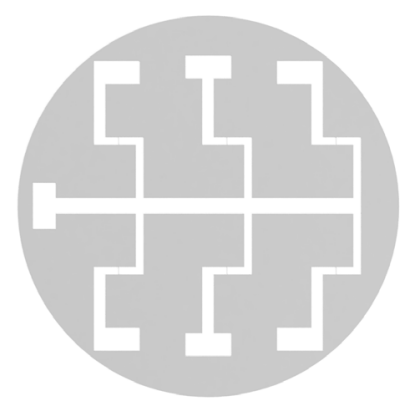

Figure 1. Negative electrode mask. Diagram of the negative electrode mask for construction of bespoke electrode dishes.

2. Construct the mounting wafer (Figure 2).

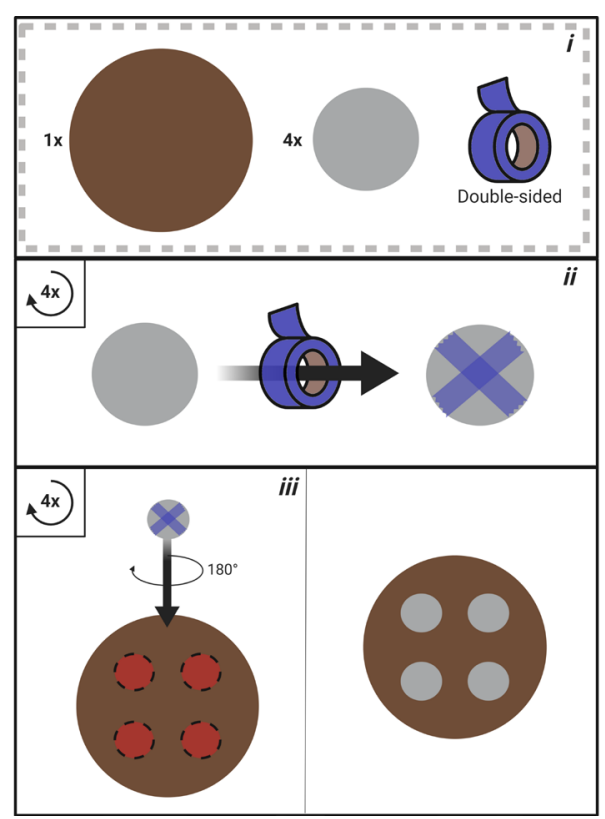

Figure 2. Mounting wafer construction process. i. Materials required for mounting wafer construction ( $1 \mathrm{x}$ stainless steel mounting wafer; $4 \mathrm{x}$ stainless steel washer; double-sided adhesive tape). ii. Apply double-sided adhesive tape to the bottom of each washer. iii. Secure each washer to the mounting wafer.

3. Place the mask into the glass-bottomed microscope dish (Figure 3). 


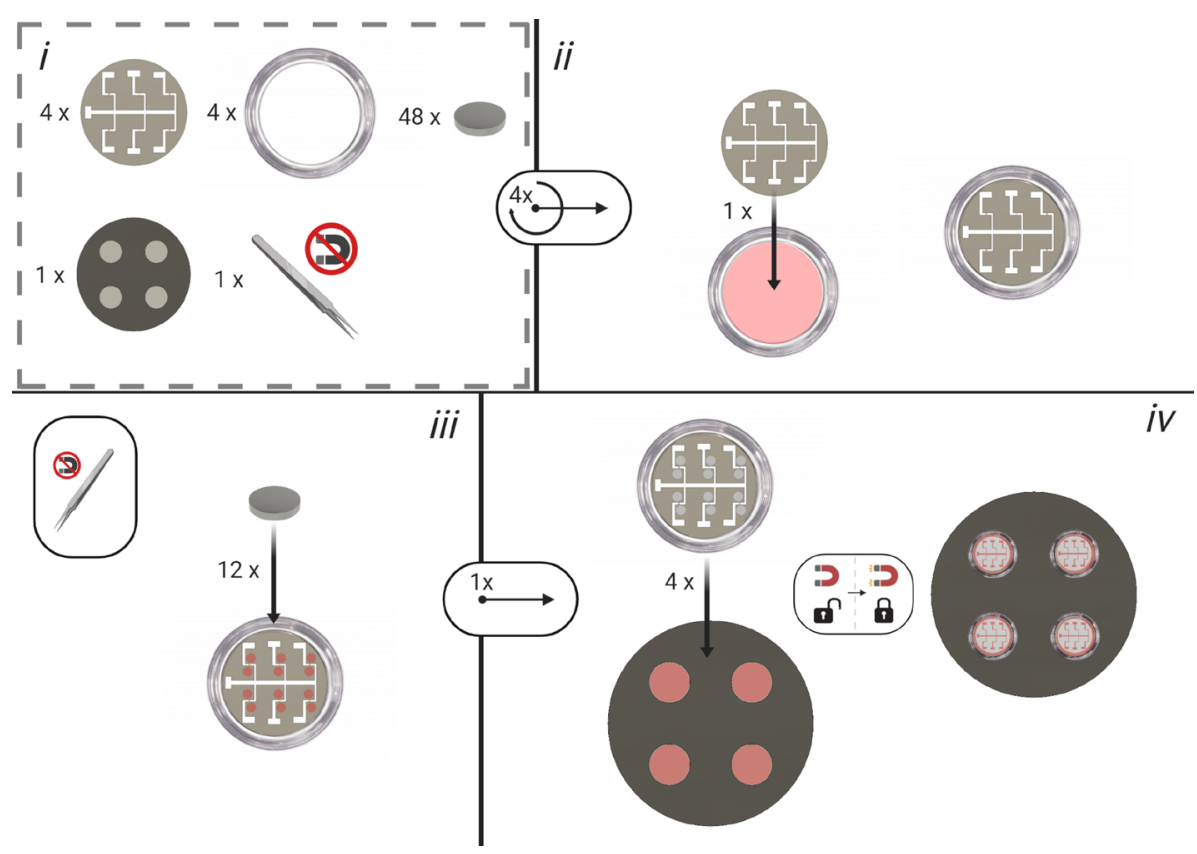

Figure 3. Bespoke Electrode Dish Construction; Steps A3-A6. All numbers correspond to their respective steps within the bespoke electrode construction process.

4. Using non-magnetic tweezers place 12 nickel magnets in positions parallel to each electrode. This ensures that the mask is in contact with the glass.

5. Mount the dish with mask, magnets and wire onto the stainless-steel platform on the mounting wafer. Place into the electron vapor deposition system.

6. Deposit the Titanium-Gold Alloy onto the dish using Electron Vapor Deposition (20 nm Ti; $80 \mathrm{~nm}$ $\mathrm{Au})$.

7. Remove the dish from the vapor deposition machine. Check the electrode gap is uninterrupted for each position using an optical microscope.

8. Solder 7 copper wires of approximately $5 \mathrm{~cm}$ to the corresponding pins of a male IDC connector.

9. Using the soldering iron, create 7 small holes in the plastic rim of the now patterned glassbottom electrode dish. Feed each of the copper wires through a hole. Secure the male IDC connector to the rim of the dish with Araldite two-part epoxy.

10. Trim the copper wires to appropriate lengths in order to contact each electrode terminal (including ground) as denoted in Figure 3. Bond the copper wire with conductive graphite epoxy. Once graphite epoxy has solidified, further coat the graphite bond with Araldite two-part epoxy for the purpose of waterproofing.

\section{B. Relay circuit construction}

The following circuit is for controlling electrical stimulation across electrodes. Individual electrodes are gated until triggered either manually or after a predetermined number of microscope images, dependent on user microscope configuration. The bill of materials may be found in Table 2. Code to run the circuit is deposited at github (github.com/ConorEd/ElectrodeStimulation). 
1. Solder two female BNC connectors; 6 Relays; $61 \mathrm{M} \Omega$ resistors and two 10-way male IDC connectors to the prototyping board, as shown in Figure 4.

2. Mount the Arduino UNO microcontroller to the prototyping board and connect the Arduino GPIO pins to the circuit as in Figure 4. This may be accomplished by either extruding breadboard jumper wires from the circuit and plugging them into the pins, or creating a 'shield' by soldering $2.54 \mathrm{~mm}$ pitch header pins to the circuit board.

3. Cut ribbon cable to appropriate lengths to reach the microscope and a signal recording device.

4. Connect two 10-way female IDC connectors to either end of the microscope cable.

5. Connect the Arduino UNO microcontroler to a computer with the USB B cable and upload the code found at www.github.com/ConorEd/ElectrodeStimulation.

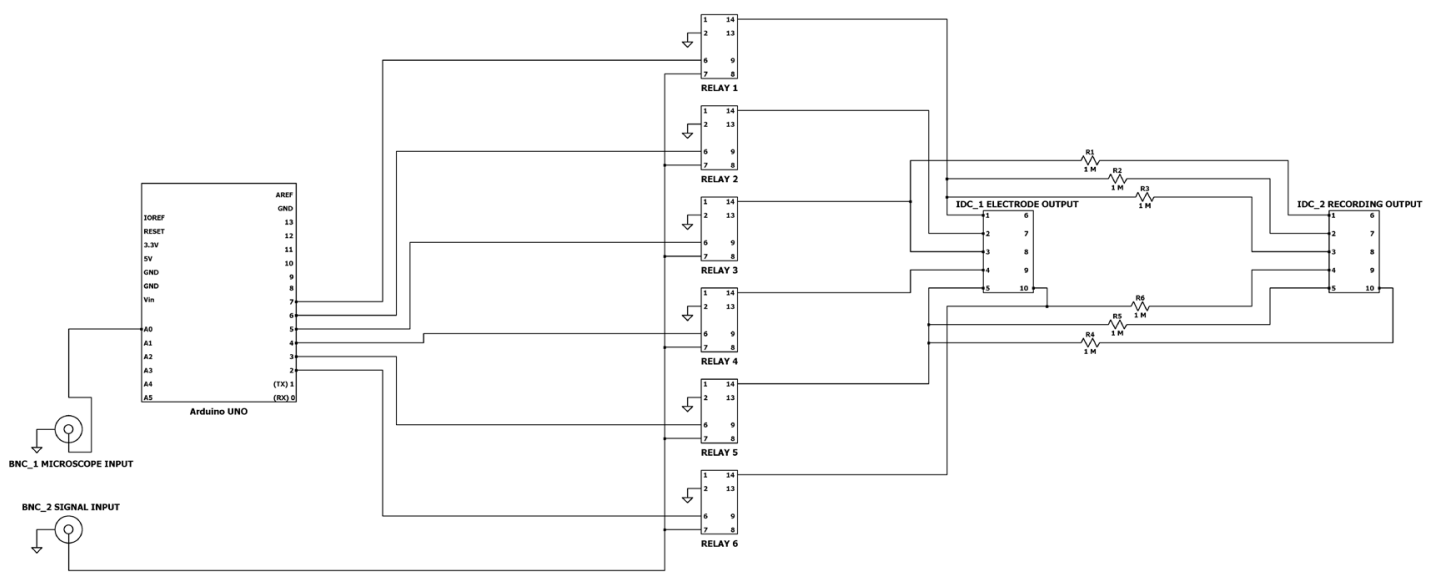

Figure 4. Relay Circuit Diagram. Detailed schematic for construction of the relay circuit.

C. Sample preparation (Figures 5 and 6 )

The method may be applied to many bacterial strains or species, subject to appropriate choice of liquid and solid growth media. Wildtype Bacillus subtilis (NCIB3610) or Escherichia coli (K12), at a density of $\mathrm{OD}_{600}=1.5$, serves as a good model organism for the method and was used in Stratford et al., 2019.

The following protocol details the procedure for a culture of wildtype $B$. subtilis (NCIB3610), OD 600 1.5. Using cultures treated with or without antibiotic vancomycin, the procedure determines between antibiotic-susceptible and -resistant cells. In Stratford et al. (2019), we showed that cells treated with UV, ethanol or CCCP show the response distinct from untreated proliferative cells. Cells treated with different antimicrobial agents or procedures can be tested for their electrical responses for rapid AST.

Note: While this method should apply to a broad range of microbes, adaptation may be required for use with species and/or strains with different growth requirements. 


\section{LB culture}

1. Place a single colony of $B$. subtilis into $5 \mathrm{ml}$ aliquots of $L B$ liquid media. Use the standard aseptic technique of microbiology.

2. Place in overnight in a shaking incubator $\left(37^{\circ} \mathrm{C} ; 200 \mathrm{rpm}\right)$. Monitor the optical density of cell cultures using a spectrophotometer, the benchmark $\mathrm{OD}_{600}$ for this procedure at the time of experimentation is approximately 1.5

\section{MSgg liquid culture}

1. Centrifuge $B$. subtilis LB cultures at $3,220 \times g(4,000 \mathrm{rpm})$ for $5 \mathrm{~min}$; retain the pellet and discard the liquid supernatant.

2. Resuspend the pellets in the equal volume of liquid MSgg media supplemented with or without vancomycin (or other antimicrobial agents).

3. Place the culture in a shaking incubator $\left(30^{\circ} \mathrm{C} ; 200 \mathrm{rpm}\right)$ for $1 \mathrm{~h}$.

\section{Creating agar pads *}

1. $\sim 30$ min prior to retrieving the cultures, create the agar pads

Place a microscope cover glass on Parafilm, this ensures the liquid MSgg agar solution remains on the cover glass. Add $1 \mathrm{ml}$ of liquid MSgg agar to a single $22 \times 22 \mathrm{~mm}$ glass slip, carefully covering the whole slip.

a. Immediately place another coverslip on top of the agar, then wait for the agar to solidify. Avoid bubbles to be formed.

b. Using tweezers and scalpel, prize the top glass slide off the solid agar, then cut the agar into $\sim 50 \mathrm{~mm}^{2}$ square pads.

D. Loading samples into electrode dish or Cytecom sample holder (Figures 5 and 6)

1. Add $1.5 \mu \mathrm{l}$ of cell suspension to each of the agar pads, ensuring the droplet is central and not touching edges of the pad, to prevent lateral pad drifts due to bacterial growth.

2. Wait $10 \mathrm{~min}$ for the drop to dry into the agar pad at room temperature.

3. Using a scalpel and tweezers, slowly lift the agar pad from the bottom and flip onto the electrode, sample side down, so as the sample is in direct contact with the electrode.*

Note: The agar pads can be very fragile, take care not to break or scratch agar pads. Make sure to place the inoculated side facing the electrode.

4. Close the lid on the dish or Cytecom sample holder to prevent sample drying.

Note: If using the Bespoke electrode dish, ensure Parafilm seals the dish shut, to prevent excessive moisture loss from the samples.

E. Preparing sample for microscopy

1. Secure the dish in the microscope stage insert. If using an oil immersion lens, add immersion oil onto the bottom of the dish or Cytecom sample holder, over each electrode. 
2. Place the stage insert onto the microscope stage.

3. Open MetaMorph (or equivalent microscope operating software).

4. Locate the position of an electrode and find the correct focus under phase contrast, DIC or brightfield illumination. Save the position of electrode using the microscope software. Repeat this for all 6 electrodes.

Note: Add a 'rest' position well away from the electrodes, this should be a place for the objective to remain away from the electrodes between imaging sequences. This helps prevent the pads from drying out.

5. Leave the sample to settle in the microscope chamber for $1 \mathrm{~h}$ at $30^{\circ} \mathrm{C}$ to prevent focus drift due to thermal shift.

F. Time-lapse microscopy settings

1. Set the microscope parameters as per Table 4. Time-lapse is taken for each position separately.

Table 4. Time-lapse Microscopy Parameters

\begin{tabular}{ll}
\hline Parameter & Setting \\
\hline Total Duration & $1 \mathrm{~min}$ \\
Image Interval & $500 \mathrm{~ms}$ \\
Wavelength(s) & $434 \mathrm{~nm}$ (CFP Filter) \\
Exposure Time & $150 \mathrm{~ms}$ \\
Fluorescent Light Intensity & $\mathrm{X}$ \\
\hline
\end{tabular}

2. Upload code (https://github.com/ConorEd/ElectrodeStimulation) to the Arduino UNO.* microcontroller, ensuring all relays are set to be closed. Either manual or microscope-triggered relay control may be set prior to upload within the Arduino IDE.

3. Verify that the electrical stimulation settings are correct (as per Table 5).

4. Turn on the signal generator.*

5. Connect the electrode dish to the device via IDC connector (Figure 7).

Table 5. Electrical stimuli parameters

\begin{tabular}{ll}
\hline Parameter & Setting \\
\hline Duration & $2,500 \mathrm{~ms}$ \\
Waveform & Sine \\
Frequency & $100 \mathrm{~Hz}$ \\
Voltage & $\pm 1.5 \mathrm{~V}$ \\
Offset & $0 \mathrm{~V}$ \\
\hline
\end{tabular}




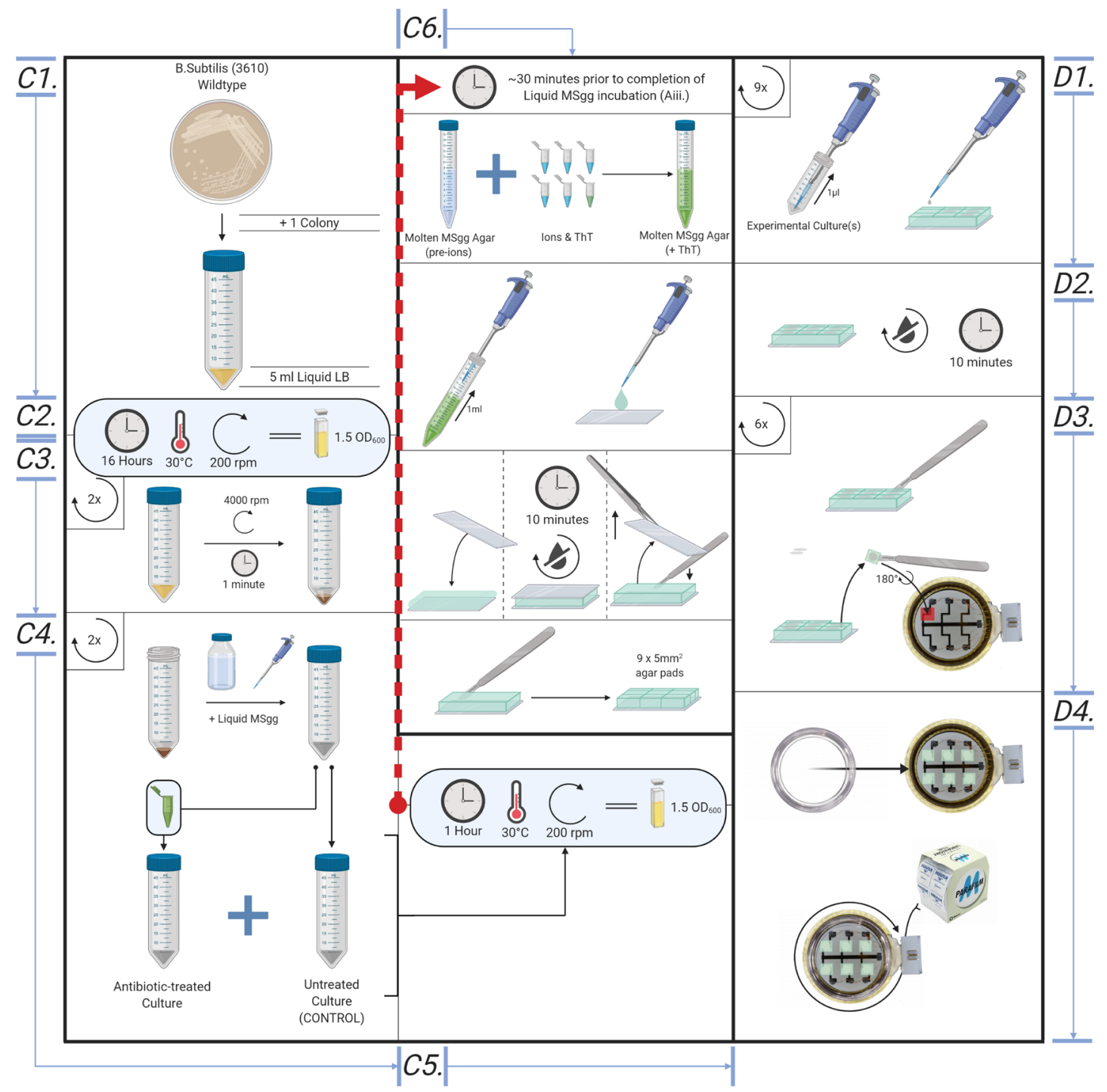

Figure 5. Overview of the bespoke protocol, from cell culture to microscope. Illustrative diagram for creating cell culture, MSgg liquid culture, creating agar pads and loading samples into Cytecom sample holder. 


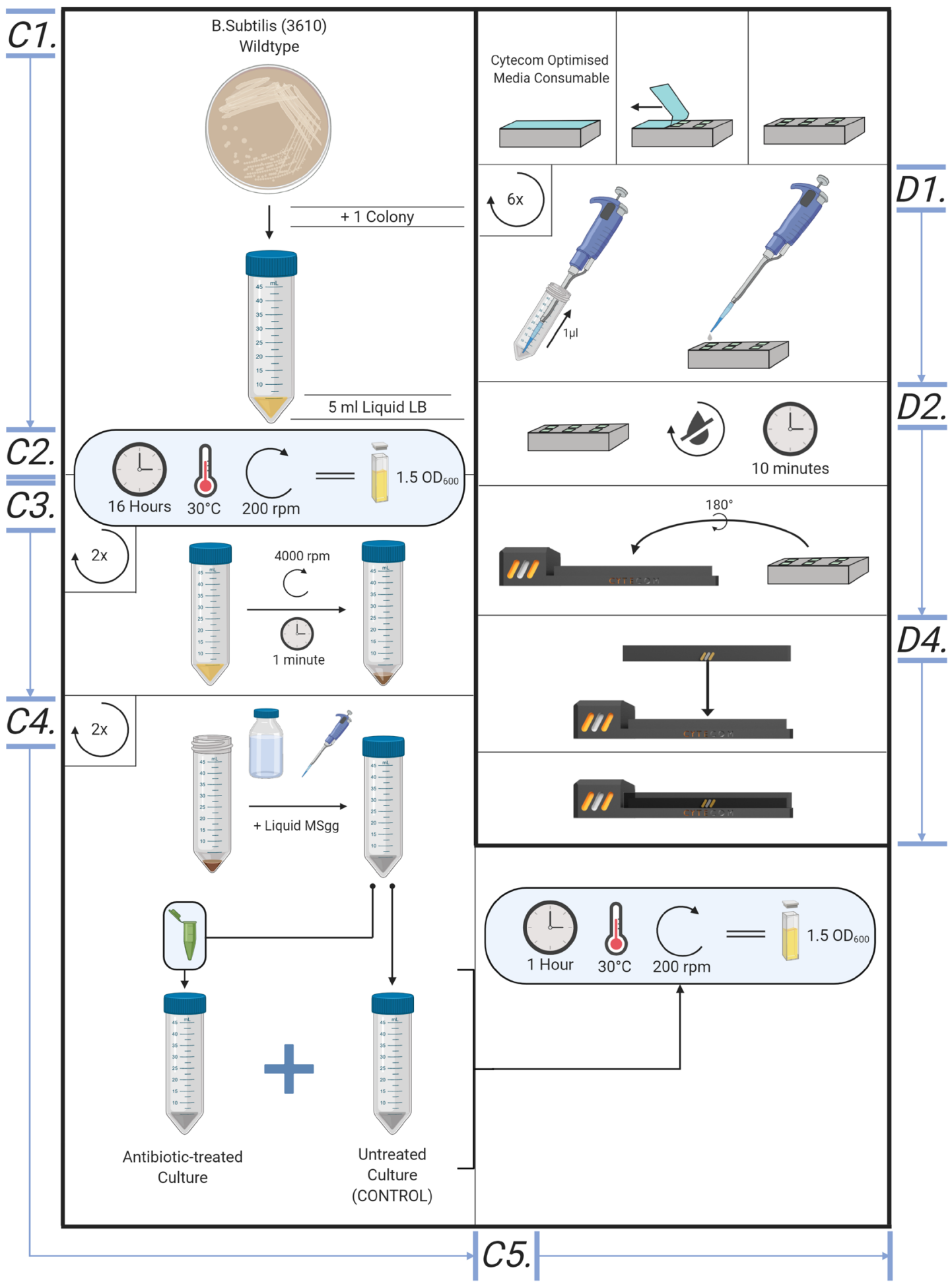

Figure 6. Overview of the cytecom protocol; from cell culture to microscope. Si. Creating Cell Cultures (Method found in supplementary information). Ci. MSgg Liquid Culture. D. Loading samples into the Cytecom sample holder. 


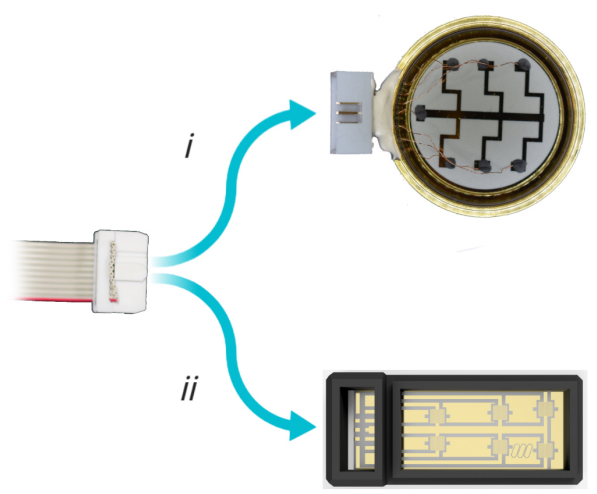

Figure 7. Connecting dish to device. Connect the signal output IDC connector to i) the bespoke dish or ii) CytePulse stage insert, respectively.

6. Start image acquisition through the microscope software.

\section{Data analysis}

1. Load the time-lapse image series in Fiji/lmageJ (Schindelin et al., 2012) (or, equilibrant image analysis software)

Note: It is advisable to play the dataset as a movie to ensure there is no focus drifting, image drifts or bubble formation by electrolysis.

2. Add regions of interest (ROIs) around individual cells using ROI manager in ImageJ.

3. Use multi-measures function in ROI manager to measure the intensity values over time. In the founding study, we observed that fluorescence intensity increases in proliferative cells while decreases in inhibited cells.

\section{$\underline{\text { Recipes }}$}

Note: All Media need to be autoclaved and treated with standard microbiology aseptic techniques.

1. $\mathrm{LB}$

Mix $25 \mathrm{~g} / \mathrm{L}$ as directed on the bottle

2. $0.5 \mathrm{M}$ potassium phosphate buffer $(\mathrm{pH} 7)$

a. Mix $68.5 \mathrm{ml} 1 \mathrm{M} \mathrm{K}_{2} \mathrm{HPO}_{4}$ and $31.5 \mathrm{ml} 1 \mathrm{M} \mathrm{KH}_{2} \mathrm{PO}_{4}$

b. Add $100 \mathrm{ml}$ of water to dilute down to $0.5 \mathrm{M}$

3. $1 \mathrm{M}$ MOPS buffer

a. Make up 1 mole MOPS salt into $900 \mathrm{ml}$ water

b. Titrate the $\mathrm{pH}$ to 7.0 using $\mathrm{NaOH}$

c. Mass up to $1 \mathrm{~L}$

4. Modified MSgg

a. $20 \mathrm{ml}$ sterile MillQ water

b. $200 \mu \mathrm{l} 1 \mathrm{M}$ MOPS buffer 
c. $200 \mu \mathrm{l} 0.5 \mathrm{M}$ potassium phosphate buffer

d. For MSgg Agar only: $0.3 \mathrm{~g}$ agar

Note: Autoclave after adding ingredients a-c [a-d in the case of solid MSgg agar]. Add ingredients e-n [e-o in the case of solid MSgg agar] just prior to use of the media in the procedure.

e. $200 \mu \mathrm{l}$ of $50 \%$ glycerol $(\mathrm{v} / \mathrm{v})$

f. $200 \mu \mathrm{l}$ of $25 \%$ glucose $(\mathrm{w} / \mathrm{v})$

g. $400 \mu \mathrm{l}$ of $25 \%$ L-Glutamic acid (w/v)

h. $200 \mu \mathrm{l}$ of $0.1 \mathrm{M}$ Ammonium chloride

i. $20 \mu \mathrm{l}$ of $0.7 \mathrm{M} \mathrm{CaCl}_{2}$

j. $20 \mu \mathrm{l}$ of $2 \mathrm{M} \mathrm{MgCl}_{2}$

k. $20 \mu \mathrm{l}$ of $0.1 \mathrm{M} \mathrm{FeCl}_{3}$

l. $20 \mu \mathrm{l}$ of $0.05 \mathrm{M} \mathrm{MnCl}_{2}$

m. $0.4 \mu$ of $0.1 \mathrm{M}$ Thiamine

n. $0.2 \mu \mathrm{l}$ of $0.1 \mathrm{M} \mathrm{ZnCl}_{2}$

o. For MSgg Agar only: $20 \mu$ of $10 \mathrm{mM}$ ThT

p. For AST, supplement with antimicrobial agents as needed

\section{Acknowledgments}

This protocol is adapted from Stratford et al., 2019. The work is supported by Biotechnology and Biological Sciences Research Council (BBSRC)/Engineering and Physical Sciences Research Council (EPSRC) grant to the Warwick Integrative Synthetic Biology Centre (Grant BB/M017982/1).

\section{Competing interests}

JPS and MA are the founder and shareholders of Cytecom Ltd (Cytecom.co.uk). JPS and CLAE are employees of Cytecom Ltd. JPS and MA have filed a patent for the reported technology.

\section{References}

1. Ahmed, A., Rushworth, J. V., Hirst, N. A. and Millner, P. A. (2014). Biosensors for whole-cell bacterial detection. Clin Microbiol Rev 27(3): 631-646.

2. Benarroch, J. M. and Asally, M. (2020). The microbiologist's guide to membrane potential dynamics. Trends in Microbiology.

3. Bruni, G. N., Weekley, R. A., Dodd, B. J. T. and Kralj, J. M. (2017). Voltage-gated calcium flux mediates Escherichia coli mechanosensation. Proc Natl Acad Sci U S A 114(35): 9445-9450.

4. Damper, P. D. and Epstein, W. (1981). Role of the membrane potential in bacterial resistance to aminoglycoside antibiotics. Antimicrob Agents Chemother 20(6): 803-808.

5. Iqbal, S. S., Mayo, M. W., Bruno, J. G., Bronk, B. V., Batt, C. A. and Chambers, J. P. (2000). $\underline{A}$ 
review of molecular recognition technologies for detection of biological threat agents. Biosens Bioelectron 15(11-12): 549-578.

6. Prindle, A., Liu, J., Asally, M., Ly, S., Garcia-Ojalvo, J. and Süel, G. M. (2015). lon channels enable electrical communication in bacterial communities. Nature 527(7576): 59-63.

7. Schindelin, J., Arganda-Carreras, I., Frise, E., Kaynig, V., Longair, M., Pietzsch, T., Preibisch, S., Rueden, C., Saalfeld, S., Schmid, B., Tinevez, J. Y., White, D. J., Hartenstein, V., Eliceiri, K., Tomancak, P. and Cardona, A. (2012). Fiji: an open-source platform for biological-image analysis. Nat Methods 9(7): 676-682.

8. Sirec, T., Benarroch, J. M., Buffard, P., Garcia-Ojalvo, J. and Asally, M. (2019). Electrical polarization enables integrative quality control during bacterial differentiation into spores. iScience 16: 378-389.

9. Strahl, H. and Hamoen, L. W. (2010). Membrane potential is important for bacterial cell division. Proc Natl Acad Sci U S A 107(27): 12281-12286.

10. Stratford, J. P., Edwards, C. L. A., Ghanshyam, M. J., Malyshev, D., Delise, M. A., Hayashi, Y. and Asally, M. (2019). Electrically induced bacterial membrane-potential dynamics correspond to cellular proliferation capacity. Proc Natl Acad Sci U S A 116(19): 9552-9557.

11. Sträuber, H. and Müller, S. (2010). Viability states of bacteria-specific mechanisms of selected probes. Cytometry A 77(7): 623-634. 